features of mechanical behaviour seem to have been explained, several new questions have emerged, and these remain unanswered. The best paper on carbon fibres-indeed of the whole conference-came from W. O. Ruland (Union Carbide), who described some impressive X-ray and electron microscope work, and presented a model for the structure of carbon fibres with which most other speakers agreed in principle. The model is of a structure composed of ribbon-like fibrils containing highly disordered graphitic planes which extend over many crystallite diameters and which are erratically stacked. The fibrils are wrinkled, and the wrinkling is greater the less the degree of preferred orientation of the basal planes.

The fibrils are not tightly bonded together because, as W. Watt (Royal Aircraft Fstablishment) suggested, the cross-linking reactions which occur between 300 and $400^{\circ} \mathrm{C}$ during pyrolysis do not cross the polymer fibril boundaries. As a result, there are many needlelike micropores some $10-20 \AA$ in diameter disposed between these fibrils. Stretching this type of structure will cause an unwrinkling of the ribbons, and lead to non-Hookean elastic behaviour of the fibres, in agreement with experimental observations described by W. N. Reynolds (Atomic Energy Research Establishment), R. Bacon (Union Carbide) and J. W. Johnson (Rolls-Royce). Speakers did not agree about the size of fibrils and micropores and the source of the pores, and the question of whether the mechanism of unwrinkling was stress-induced orientation and motion of crystallite boundaries or the movement of dislocations was unresolved.

The second day was devoted to fibres and whiskers other than carbon. Possibly the greatest interest was in the manufacture and properties of the larger diameter ceramic fibres, which can be continuously produced, and their compatibility with high-temperature metallic matrix materials. The most interesting disagreement of the day (for onlookers at least) was about the question of whether or not the boron fibre material was amorphous or crystalline. The inevitable question of the economics of boron and carbon fibres was raised, but left unresolved. A potentially exciting means of achicving a higher elastic modulus in oriented polyethylene by chain-extension during high pressure erystallization treatments was described by $\mathrm{D}$. C. Bassett (University of Reading), and the conference was rounded off by G. F. Heron (Turner Brothers Asbestos) discussing the great possibilities available to users of the cheapest material available to composite manufacturers-asbestos. As he obscrved, this is one of the oldest of the reinforcing fibres, having been used by the Greeks for reinforeing ceramics, but its potential as a competitor to glass and the new and expensive fibres is still far from being realized.

In general terms the conference was successful, but some of the most important questions were not asked. Even if they were, the frequent, irritating response "I am not allowed to answer that" inhibited further comment.

\section{MOLECULAR BEAMS}

\section{Chemical Accelerators}

\section{from a Correspondent}

T'HF investigation of chemical change is moving nearer the ideal experiment in which the reactants in specific quantum states collide with a well defined energy, and the distribution of products by chemical species, quantum state, velocity and scattering angle are measured. This objective is being pursued in several laboratories with chemical accelerators-a term which came into use at the conference of this title in Boulder, Colorado, last year (Science, 162, 818; 1968), and used to describe devices in which beams of chemically interesting species interact. In principle, the method is similar to nuclear accelerator experiments, although the actual techniques are very different. Particular difficulties arise in producing beams in the chemical energy range of less than a tenth to several hundred $\mathrm{eV}$, and in detecting the very low concentrations of product which are formed.

One approach to these problems has been described by Menzinger and Wolfgang ( $J$. Chem. Phys., 50, 2991; 1969) whose experiments with the accelerator ADAM II have reached a half way stage. By combining beam techniques with isotopic labelling gas chromatography and radio counting, and using interpretative methods developed for nuclear recoil (hot atom) chemistry, they have been able to study the reaction of tritium with cyclohexane in the energy range 1 to $200 \mathrm{eV}$. In their latest experiments $\mathrm{T}^{+}$and $\mathrm{T}_{2}{ }^{+}$are produced in a plasma source, extracted into a mass analyser and then decelerated in a multi-element lens to produce an ion beam with an energy variable between 1 and $200 \mathrm{eV}$, and with an encrgy spread of $0 \cdot 1$ to $0 \cdot 2 \mathrm{eV}$. The ion beam finally strikes a target of solid cyclohexane which is continuously deposited from a cyclohexane beam onto a surface cooled by liquid nitrogen. At the target surface charge neutralization forms ' $\mathrm{T}$ ' and ' $\mathrm{T}_{2}$ with essentially unchanged kinetic energy. The resulting hot atom then undergoes collisions in the bulk of the cyclohexane in which energy loss or chemical reaction can occur in the same way as for hot atoms produced more conventionally. After about twenty minutes the target is removed to a radio gas chromatograph and the yields of tritiated cyclohexane, n-hexane and hexenes are estimated. For the cyclohexane ' $\mathrm{T}$ and $\mathrm{n}$-hexane $\mathrm{T}$ products which correspond to processes in which a $\mathrm{C}-\mathrm{H}$ and a $\mathrm{C}-\mathrm{H}+\mathrm{C}-\mathrm{C}$ bond are ruptured, threshold energies of 1.5 and $4.5 \mathrm{eV}$ were observed. From a more detailed analysis it was also possible to estimate total reaction cross-sections as a function of energy.

A rather different technique has been used by Herschbach and his colleagues at Harvard (J. Chem. Phys., 49, 2447; 1968) to study reactions such as $\mathrm{Cl}+\mathrm{Br}_{2} \rightarrow \mathrm{ClBr}+\mathrm{Br}$. In their apparatus the beams are produced by hydrodynamic flow from Laval nozzles (Anderson, Andres and Fenn, Adv. Chem. Phys., 2\%5, $10 ; 1966)$. Beam energies from nozzles are less easily varied and have a more restricted energy range than for the ion neutralization technique. On the other hand, the intensities can be very much higher so that in the experiments at Harvard it was possible to use two intersecting beams and to detect the product scattered from the beam collision zone using a mass spectrometer which rotated about the collision centre. With this configuration the product scattering angle and the collision energy are directly measurable and information on the molecular dynamies of the reaction can be obtained more directly than from the solid target experiments. It scems likely that both techniques will contribute importantly to our understanding of the detailed mechanics of chemical reaction. 